\title{
Expression of cortactin in human gliomas and its effect on migration and invasion of glioma cells
}

\author{
LEILEI WANG $^{1}$, KAI ZHAO ${ }^{1}$, BINGCHENG REN ${ }^{2}$, MENG ZHU $^{1}$, CHEN ZHANG $^{1}$, \\ PENGFEI ZHAO $^{1}$, HUA ZHOU ${ }^{1}$, LEI CHEN ${ }^{1}$, SHENPING YU ${ }^{2}$ and XUEJUN YANG ${ }^{1,2}$ \\ ${ }^{1}$ Tianjin Neurological Institute; ${ }^{2}$ Department of Neurosurgery, Tianjin Medical University \\ General Hospital, Tianjin 300052, P.R. China
}

Received March 27, 2015; Accepted June 25, 2015

DOI: $10.3892 /$ or.2015.4156

\begin{abstract}
The aim of the present study was to investigate the role of cortactin in the infiltrative behavior of glioma cells and the potential mechanism of cortactin in promoting the migration and invasion of glioma cells. The expression of cortactin was detected by immunohistochemistry in 40 human glioma specimens and 8 non-tumor brain specimens. U251, LN229 and SNB19 glioma cells were employed for the in vitro study and assigned into the siRNA-cortactin (transfected with siRNA specific to cortactin), siRNA-NC (transfected with negative control RNA sequence) and siRNA-N (transfected with empty vector) groups. The expression of cortactin in different treated glioma cell groups was detected using western blot analysis and RT-qPCR. The migration and invasion of glioma cells under different treatments were assessed using a wound-healing assay and Transwell-chamber invasion assay, respectively. The lamellipodia of glioma cells following treatment were observed by immunofluorescence (IF) and changes of lamellipodia over time were imaged using an inverted microscope. The distribution of cortactin and the actin-related protein 2/3 (Arp2/3) complex in glioma cells were observed after IF detection. The expression of cortactin in the glioma specimens was significantly higher than that in non-tumor brain tissue $(\mathrm{P}<0.05)$ and positively correlated with the malignancy of glioma specimens $(r=0.912, \mathrm{P}=0.00)$. The cortactin expression in glioma cells was markedly inhibited $(\mathrm{P}<0.05)$ and their migration and invasion ability was also impaired significantly following treatment with siRNA $(\mathrm{P}<0.05)$ compared with the other two groups. The size and persistence time of lamellipodia were reduced after cortactin expression was inhibited in glioma cells. Cortactin and the Arp2/3 complex were co-localized in the front of glioma cells, where actin was polymerized and lamellipodia formed. Thus,
\end{abstract}

Correspondence to: Dr Xuejun Yang, Department of Neurosurgery, Tianjin Medical University General Hospital, No. 154, Anshan Dao Road, Tianjin 300052, P.R. China

E-mail: ydenny@yahoo.com

Key words: glioma, cortactin, migration, invasion, lamellipodia, Arp2/3 complex the results revealed that, cortactin is crucial in invasion and migration of glioma cells, which may promote the migration and invasion of glioma cells by regulating lamellipodia formation, a process requiring the combination of cortactin and the Arp2/3 complex.

\section{Introduction}

Malignant gliomas are the most common primary malignant tumors in brain. Although various therapeutic modalities are available, the disease remains incurable. The median survival rate in patients with newly diagnosed glioblastoma multiforme is $\sim 15$ months even when treated by surgery alone or combined with radiotherapy and chemotherapy (1-3). The most important reason for the failure of clinical management is the infiltrative and migrating behavior of gliomas, which leads to diffuse growth and/or recurrence of the tumor (4). Therefore, new therapeutic strategies are required to prevent the invasion and migration of glioma cells effectively.

The regulation of cell migration is critical to normal and pathological processes, including development, immune function, such as neutrophils and macrophages and tumor metastasis (5). Lamellipodia have been proven essential for the directional migration of cells (6). The formation of lamellipodia depends on a highly branched network of polymerized actin filaments at the barbed ends, which drives membrane extension at the leading edge of cells (7). The actin-related protein $2 / 3$ (Arp2/3) complex is a key regulator of the actin network. It binds to the side of a pre-existing filamentous $(\mathrm{F})$-actin filaments and stimulates new filament formation to create branched actin networks, a process termed the 'dendritic nucleation' model of cortical actin assembly (8-10). Previous findings confirmed the role of the Arp2/3 complex in the metastasis of many tumors, including glioma. The ability of migration and invasion of tumor cells may be significantly reduced after Arp $2 / 3$ complex disruption by RNA interference (11-13).

Cortactin is an Arp2/3 complex-activating and F-actinbinding protein. It possess a multi-domain structure consisting of an acidic domain at the amino terminus (NTA), followed by six complete and one partial tandem repeating segments, a proline-rich helical region and an Src homology $\mathrm{SH}_{3}$ domain located at the carboxyl terminus $(14,15)$. Cortactin was first identified as a prominent substrate of the 
Src non-receptor tyrosine kinase. Subsequently, cortactin has been shown to play an essential role in many actin-based cell processes, including migration and invasion, axon guidance, neuronal morphogenesis and tumor cell metastasis (15). In many of the processes, cortactin regulates activation of the Arp2/3 complex and stabilizes actin branch points in the dynamic assembly and disassembly of actin polymerization at the cell periphery. The ability of cortactin to promote actin polymerization requires the NTA domain, which binds the Arp3 subunit of the Arp2/3 complex. Cortactin lacking the NTA domain fails to localize at the cell periphery. The $\mathrm{SH}_{3}$ domain of cortactin regulates its ability to activate the Arp2/3 complex synergized with some proteins, including WiskottAldrich syndrome protein (WASP) and WASP-interacting protein (WIP) (16-18).

As mentioned above, cortactin facilitates migration by increasing lamellipodia persistence and promotes adhesion assembly through the binding and activation of the Arp $2 / 3$ complex. In previous studies, amplification of segment 11q13 on chromosome 11, a region that includes the CTTN gene associated the over expression of cortactin, has been associated with many types of cancer, including oral squamous and head and neck squamous cell carcinoma (HNSCC), lung, breast, colorectal cancer and melanoma (15,19-22) However, whether cortactin also plays a role in migration and invasion of glioma cells remains to be determined. In the present study, we investigated cortactin expression in human gliomas with different WHO grade and how cortactin influenced the morphology and motility of glioma cells by regulating the formation of lamellipodia.

\section{Materials and methods}

Reagents and specimens. Cortactin antibody (Abcam, Burlingame, CA, USA); p34-Arc antibody (Millipore, Billerica, CA, USA), which was specific for the Arp2/3 complex; rhodamine phalloidin (Invitrogen-Life Technologies, Carlsbad, CA, USA) used for actin staining; Alexa Fluor 488 and 555-conjugated and Texas Red goat anti-mouse secondary antibodies (Invitrogen-Life Technologies); Triton X-100 (Solarbio, Haidian, Beijing, China); 4\% paraformaldehyde (Solarbio), DAPI (Sigma, St. Louis, MO, USA); and Lipofectamine ${ }^{\mathrm{TM}}$ 2000 Reagent (Invitrogen) were used in the present study.

Forty tumor specimens were obtained from patients with glioma by surgical resection at the Department of Neurosurgery, Tianjin Medical University General Hospital (Tianjin, China) from July 2011 to November 2013. None of the patients had undergone radiation or chemotherapy prior to surgical therapy. The pathological diagnosis and grading for each glioma was assessed by neuropathologists according to the 2007 World Health Organization (WHO) Classification of Nervous System Tumors. Glioma specimens included 6 cases of diffuse astrocytoma (WHO grade II), 9 of oligoastrocytoma (WHO grade II), 9 of anaplastic oligodendroglioma (WHO grade III) and 16 of glioblastoma (WHO grade IV). Eight specimens of non-tumor brain tissues were obtained from patients undergoing craniotomy for epilepsy as the control.

Immunostaining results were determined using 5 high power fields of the specimens. To determine the intensity of the immunohistochemical staining, scores were determined as: - (negative staining for target cells) + (positive staining for $1-9 \%$ target cells), ++ (positive staining for $10-49 \%$ target cells) and +++ (positive staining for $>49 \%$ target cells). Thus, + represented weak staining, ++ was moderate staining, +++ was strong staining and - was negative staining.

The tissue samples were collected in accordance with the institutional review board-approved protocols. After surgical resection, tissue specimens were immediately frozen and stored in liquid nitrogen until use. The present study was approved by the Ethics Committee of the institutional review boards of Tianjin Medical University General Hospital. Written informed consent was obtained from all patients.

Cell culture. Human U251, LN229 and SNB19 glioma cell lines, were purchased from the Chinese Academy of Sciences Cell Bank. U251, LN229 and SNB19 cells were cultured in Dulbecco's modified Eagle's medium (DMEM) supplemented with $10 \%$ fetal bovine serum (FBS) (Solarbio) and maintained at $37^{\circ} \mathrm{C}$ in an atmosphere of $5 \% \mathrm{CO}_{2}$ and routinely passaged at 2-3 day intervals.

Immunohistochemistry. For immunohistochemistry, tissue sections were incubated with cortactin primary antibody (Abcam, 1:100 dilution) overnight at $4^{\circ} \mathrm{C}$. Biotinylated secondary antibody at a dilution of 1:100 was then added at room temperature for $30 \mathrm{~min}$, followed by incubation with ABC-peroxidase for an additional $30 \mathrm{~min}$. After washing with Tris-buffer, the sections were incubated with 3,3'-diaminobenzidine (DAB, $30 \mathrm{mg}$ dissolved in $100 \mathrm{ml}$ Tris-buffer containing $0.03 \% \mathrm{H}_{2} \mathrm{O}_{2}$ ) for $5 \mathrm{~min}$, rinsed in water and counterstained with hematoxylin.

RNA interference. RNA interference reagent (GenePharma, Hi-Tech Park, Shanghai, China) include cortactin-siRNA sequence (5'-CAAGCUUCGAGAGAAUGUCUUTT-3') and negative control sequence (5'-UUCUCCGAACGUGUCACG UTT-3'). The two type siRNA sequence dissolved in DEPC water, respectively. Cortactin-siRNA sequence $(5 \mu \mathrm{l}), 5 \mu \mathrm{l}$ negative control sequence or equal empty vector was mixed with $5 \mu \mathrm{l}$ transfection Lipofectamine ${ }^{\mathrm{TM}} 2000$ reagent in $500 \mu \mathrm{l}$ serum-free medium for the siRNA-cortactin group, siRNA-NC and siRNA-N groups, respectively.

$R T-q P C R$. The different treated glioma cells were lysed and RNA extracts were collected after $48 \mathrm{~h}$. Total RNA was isolated using the RNeasy kit (Tiangene Biotech Co., Ltd., Beijing, China). Reverse transcription-PCR (RT-PCR) reaction was implemented using the RT-PCR kit.

PCR amplification was performed under the conditions: Denaturation at $94^{\circ} \mathrm{C}$ for $5 \mathrm{~min}, 94^{\circ} \mathrm{C}$ for $30 \mathrm{sec}$, annealing at $49^{\circ} \mathrm{C}$ for $30 \mathrm{sec}$, total of 35 cycles; with a final extension at $72^{\circ} \mathrm{C}$ for $5 \mathrm{~min}$. Primer sequences for cortactin used were: forward, 5'-GAACAAGACCGAATGGATAAG-3' and reverse, 5'-TTCAAAGCCTACAGCAGAC-3'. Glyceraldehyde3-phosphate dehydrogenase (GAPDH) served as the internal standard, with the annealing temperature at $54^{\circ} \mathrm{C}$, whereas the other conditions were identical to the previous ones. Primer sequences used for GAPDH were: forward, 5'-TCTCTG CTCCTCCTGTTC-3' and reverse 5'-ATCCGTTGACTCCGA CCT-3'. 
Western blot analysis. For each specimen, $50 \mathrm{mg}$ of tissue was dissected into small sections and transferred into a $1.5 \mathrm{ml}$ microcentrifuge tube. A total of $500 \mu \mathrm{l}$ cell lysis buffer was added to the tube. The tissue was homogenized on ice with 10-15 strokes (3-4 sec/stroke) of a mini-homogenizer and plastic pestle. The sample was centrifuged at $12,000 \mathrm{x} \mathrm{g}$ for $15 \mathrm{~min}$ at $4^{\circ} \mathrm{C}$ and the supernatant was transferred to a fresh tube. A total of $50 \mu \mathrm{g}$ protein and an equal volume of $2 \mathrm{X}$ sample buffer were heated at $94^{\circ} \mathrm{C}$ for $5 \mathrm{~min}$.

Following treatment of glioma cells for $48 \mathrm{~h}$, blots of whole-cell lysates were prepared. Briefly an equal number of cells were directly lysed in SDS-PAGE loading buffer [0.1 mol/1 Tris (pH 6.8), 20\% SDS, $0.2 \%$ glycerol, $0.2 \mathrm{~mol} / 1$ DTT] and boiled at $94^{\circ} \mathrm{C}$ for $5 \mathrm{~min}$.

Proteins were separated on $10 \%$ SDS-polyacrylamide gel and then transferred onto a polyvinylidene difluoride (PVDF) membrane. The blot was blocked in PBST and 5\% skimmed dried milk at $37^{\circ} \mathrm{C}$ for $1 \mathrm{~h}$. The membrane was then incubated in primary antibody (cortactin, rabbit, $1: 1,000$ ) at $4^{\circ} \mathrm{C}$ overnight, followed by treatment with mouse anti-rabbit secondary antibody $(1: 5,000)$. Blots were developed using enhanced chemiluminescence (ECL) reagents (Amersham Pharmacia, Buckinghamshire, UK) and visualized using the Gene Genius Imaging System (Frederick, MD, USA). Cortactin antibody was used to detect the expression of cortactin and $\beta$-actin was used as the internal standard.

Wound-healing and Transwell invasion assays. For the wound-healing assay, the glioma cells of the treated groups were seeded in 6-well plates at a density of $2.0 \times 10^{5}$ cells $/ \mathrm{ml}$ and allowed to reach confluency. A confluent monolayer was obtained and wounds were created using a $200 \mu 1$ sterile pipette tip. Subsequently, cell debris was removed by washing the plates twice with PBS and fresh DMEM supplemented with $3 \%$ FBS was added to each well. The cells were then cultivated for up to $24 \mathrm{~h}$. The wound-healing area was recorded by taking photomicrographs at different time-points.

For the Transwell invasion assay, after the glioma cells were treated for $24 \mathrm{~h}$, the top chambers were coated with a layer of $25 \mathrm{mg} / \mathrm{cm}^{2}$ matrigel (Millipore). Cells $\left(5.0 \times 10^{4}\right)$ in serum-free medium were seeded in each chamber for each group after the Matrigel freezing. Serum medium $(500 \mu \mathrm{l})$ was added in the lower chambers as a chemoattractant. Following incubation for $48 \mathrm{~h}$, non-invading cells were removed from the top chamber with a cotton swab. The cells on the lower surface were fixed by replacing the culture medium in the bottom with $4 \%$ paraformaldehyde. After fixation for $15 \mathrm{~min}$ at room temperature, the chambers were rinsed in PBS and stained with $0.2 \%$ crystal violet for $10 \mathrm{~min}$. For each experimental condition, 10 image fields were photographed and quantified.

Immunofluorescence. The glioma cells under different treatments were grown on glass coverslips for $24 \mathrm{~h}$. The cells were washed and then fixed with $4 \%$ paraformaldehyde for $25 \mathrm{~min}$. Fixed cells were permeabilized by treatment with $0.5 \%$ TritonX-100 for $5 \mathrm{~min}$ and blocked by incubation with 5\% BSA in PBS for $1 \mathrm{~h}$. The cells were then incubated overnight at $4^{\circ} \mathrm{C}$ with cortactin (rabbit) and p34-Arc (mouse) antibodies at a dilution of 1:100. The cells were washed three times with PBS and then incubated for $1 \mathrm{~h}$ with Alexa 488-conjugated
Table I. Comparison of cortactin staining intensity in different pathological grade gliomas and non-tumor brain tissue (Case).

\begin{tabular}{lrrrrr}
\hline & & \multicolumn{4}{c}{ Results } \\
\cline { 3 - 6 } $\begin{array}{l}\text { Tissue } \\
\text { type }\end{array}$ & $\begin{array}{c}\text { Sample } \\
\text { cases }\end{array}$ & - & + & ++ & +++ \\
\hline Control brain tissue & 8 & 8 & 0 & 0 & 0 \\
WHO II grade & 15 & 0 & 12 & 3 & 0 \\
WHO III grade & 9 & 0 & 0 & 4 & 5 \\
WHO IV grade & 16 & 0 & 0 & 2 & 14 \\
Total & 48 & 8 & 12 & 9 & 19 \\
\hline
\end{tabular}

We collected 48 sample cases for study. The 48 samples include eight non-tumor brain tissue cases, 15 WHO II grade, 9 WHO III grade and 16 WHO IV grade glioma tissue cases. The result shows cortactin expressed as: control brain tissue (-) 8 cases, WHO II grade glioma tissue (+) 12 and $3(++)$ cases ,WHO III grade glioma tissue (++) 4 and (+++) 5 cases, WHO IV grade glioma tissue (++) 2 and (+++) 14 cases. Statistical analysis demonstrated the expression of cortactin in the glioma specimens was significantly higher than in non-tumor brain tissue and positively correlated with the malignancy of glioma specimens $(\mathrm{F}=41.16, \mathrm{P}=0.00)$

goat anti-rabbit secondary antibody at a dilution of 1:1,000 for $1 \mathrm{~h}$ at $37^{\circ} \mathrm{C}$. The cells were washed with PBS and then counterstained with rhodamine phalloidin for $20 \mathrm{~min}$ to stain actin filaments and DAPI to stain DNA. The cells were imaged under a confocal microscope (Olympus FV1000; Research Center of Basic Medical Science of Tanjin Medical University Olympus, Tokyo, Japan).

Statistical analysis. Data were analyzed using SPSS 17.0 software (Chicago, IL, USA). One-way analysis of variance (ANOVA), least significant difference and Pearson's correlation tests were used. Values are presented as means \pm standard error of measurement (SEM). $\mathrm{P}<0.05$ was considered statistically significant. In vitro experiments were repeated three times.

\section{Results}

Expression of cortactin in human glioma specimens. Histological assessment for glioma specimens and normal brain tissues was performed by neuropathologists. To determine cortactin expression in human gliomas, immunohistochemistry and western blot analysis were performed. Immunohistochemistry of the tissue sections revealed that cortactin was localized at the cell cytoplasm and its signal intensity increased with the increasing tumor malignancy (Fig. 1A). A comparison of cortactin staining intensity in different pathological grade gliomas and non-tumor brain tissue is shown in Table I. Relative to $\beta$-actin, the level of cortactin in tissue specimens was $18.20 \pm 2.52 \%$ in non-tumor brain tissue (NB, $n=8), 36.20 \pm 3.34 \%$ in WHO grade II $(n=15)$, $51.40 \pm 4.3 \%$ in WHO grade III $(n=9)$ and $59.6 \pm 4.31 \%$ in WHO grade IV $(n=16)$ (Fig. 1B and C). The expression of cortactin in the glioma specimens was significantly higher than in nontumor brain tissue $(\mathrm{P}<0.05)$ and positively correlated with the malignancy of glioma specimens $(\mathrm{r}=0.912, \mathrm{P}=0.00)$. 


\section{A $\quad \mathrm{NB}$}
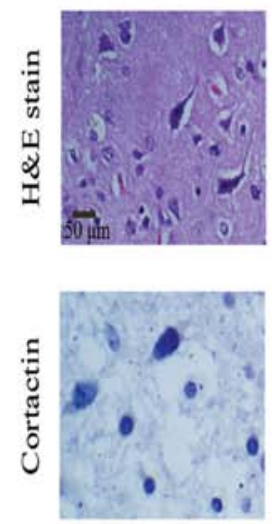

Grade II
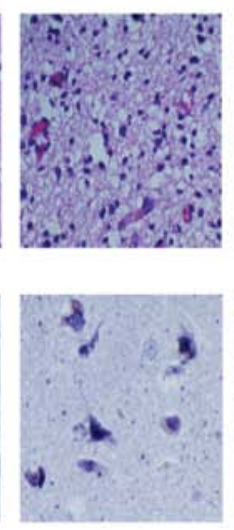

Grade III
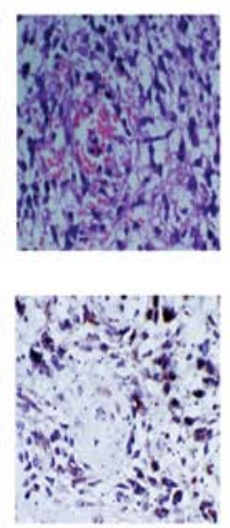

Grade IV
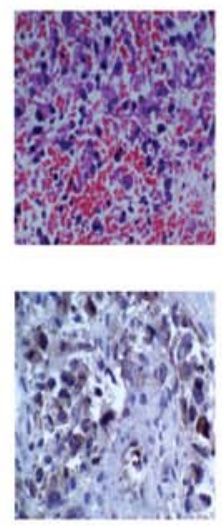

B
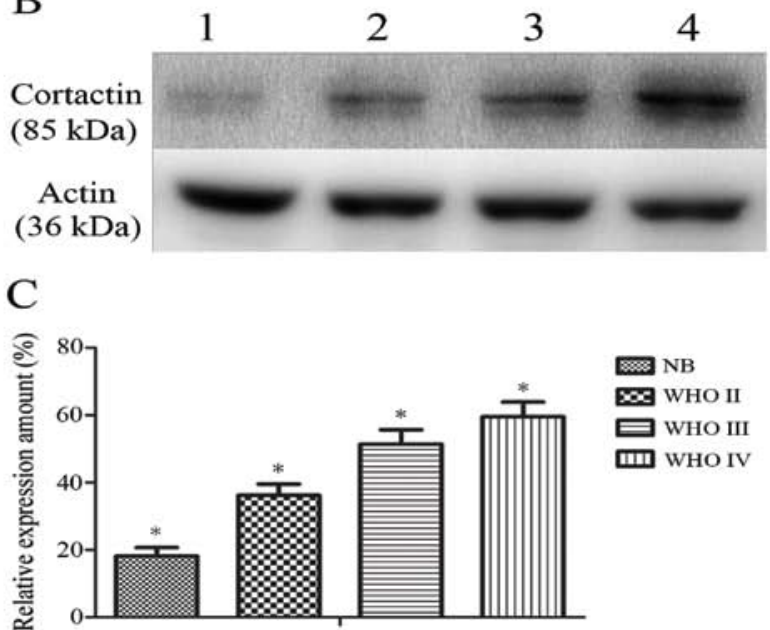

Figure 1.Expression of cortactin in human glioma specimens.(A) H\&E staining is one of the indicators of the WHO grading of the tumors. Immunohistochemistry was performed and the cells observed under a microscope. The brown stain revealed cortactin is located in the cell cytoplasm and positively correlated with the malignancy of glioma. Nuclear (blue), Cortactin (brown). (B) Western blot analysis was performed on different grades of human glioma specimens (P<0.05). (C) The relationship between the expression of cortactin and the malignancy of glioma specimens analysed using the Pearson's correlation test show a positive correlation between cortactin expression and the malignancy of glioma ( $\mathrm{r}=0.912, \mathrm{P}=0.00)$. H\&E, hematoxylin and eosin; WHO, World Health Organization; NB, non-tumor brain tissue.

Effect of cortactin-siRNA on the expression of cortactin in glioma cells. For the RT-qPCR, the expression of cortactin in glioma cells was inhibited significantly in the siRNA-cortactin group compared to the siRNA-N and siRNA-NC groups. The cortactin expression of the siRNA-cortactin group in the

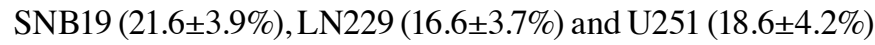
glioma cell lines was markedly decreased compared to the siRNA-N and siRNA-NC groups $(\mathrm{P}<0.05)$ (Fig. 2B). The expression of cortactin between the siRNA-NC and siRNA-N groups indicated no significant differences $(\mathrm{P}>0.05)$.

The western blot analysis revealed that for all three glioma cell lines, the expression of cortactin was significantly knocked down in the siRNA-cortactin group compared to the siRNA-N and siRNA-NC groups. Compared to $\beta$-actin, the level of cortactin in the siRNA-cortactin group in SNB19 $(15.00 \pm 1.14 \%), \mathrm{LN} 229(13.00 \pm 1.58 \%)$ and U251 $(13.80 \pm 1.66 \%)$ glioma cells was significantly decreased compared to the siRNA-N and siRNA-NC groups $(\mathrm{P}<0.05)$. The expression of cortactin between the siRNA-NC and siRNA-N groups indicated no significant differences ( $\mathrm{P}>0.05$ ) (Fig. 2A).

Knocked down expression of cortactin reduces motility of human glioma cells. The wound-healing assay was one of the first methods to be developed to study cell migration in vitro. Although not an exact duplication of cell migration in vivo, this method mimics to some extent the migration of cells in wound healing. To assess the inhibition of cortactin by siRNA on migration, we performed the assay in the different treated groups of human glioma cells. Wound closure was monitored by capturing photomicrographs at 0 and $24 \mathrm{~h}$ after wound creation. The result showed that the wound-healing area in the siRNA-cortactin group $(16.80 \pm 1.53$ in SNB19, $19.80 \pm 1.77$ in LN229 and $20.60 \pm 1.21 \%$ in U251 cells) was smaller than that in the siRNA-NC group $(52.60 \pm 2.84$ in SNB19, 62.80 \pm 3.14 in LN229 and $56.00 \pm 3.21 \%$ in U251 cells) and the siRNA-N group $(52.00 \pm 3.11$ in SNB19, 63.80 \pm 2.75 in LN229 and

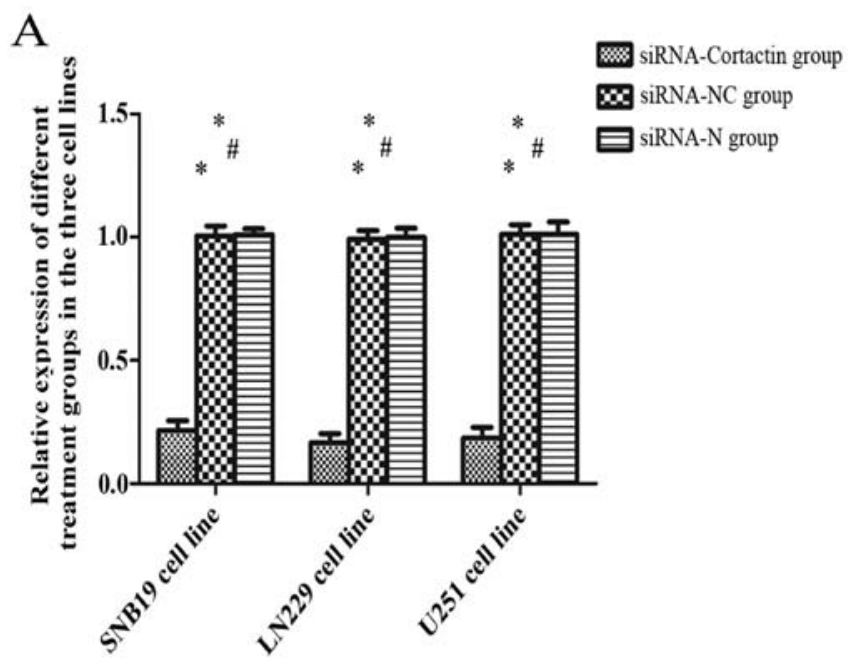

B

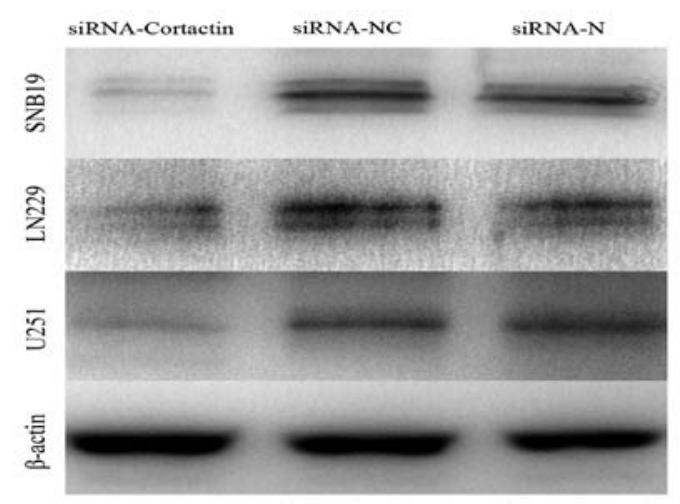

Figure 2. Expression of cortactin in different treatment groups glioma cells. (A) RT-qPCR showed that compared with the other two groups, the expression of cortactin in the siRNA-Cortactin group was significantly decreased $\left({ }^{*} \mathrm{P}<0.05\right.$ and $\left.{ }^{*} \mathrm{P}>0.05\right)$. (B) Western blot analysis shows cortactin was knocked significantly down in the Cortactin-siRNA group compared to the Cortactin- $\mathrm{N}$ and Cortactin-NC groups $(\mathrm{P}<0.05)$. 

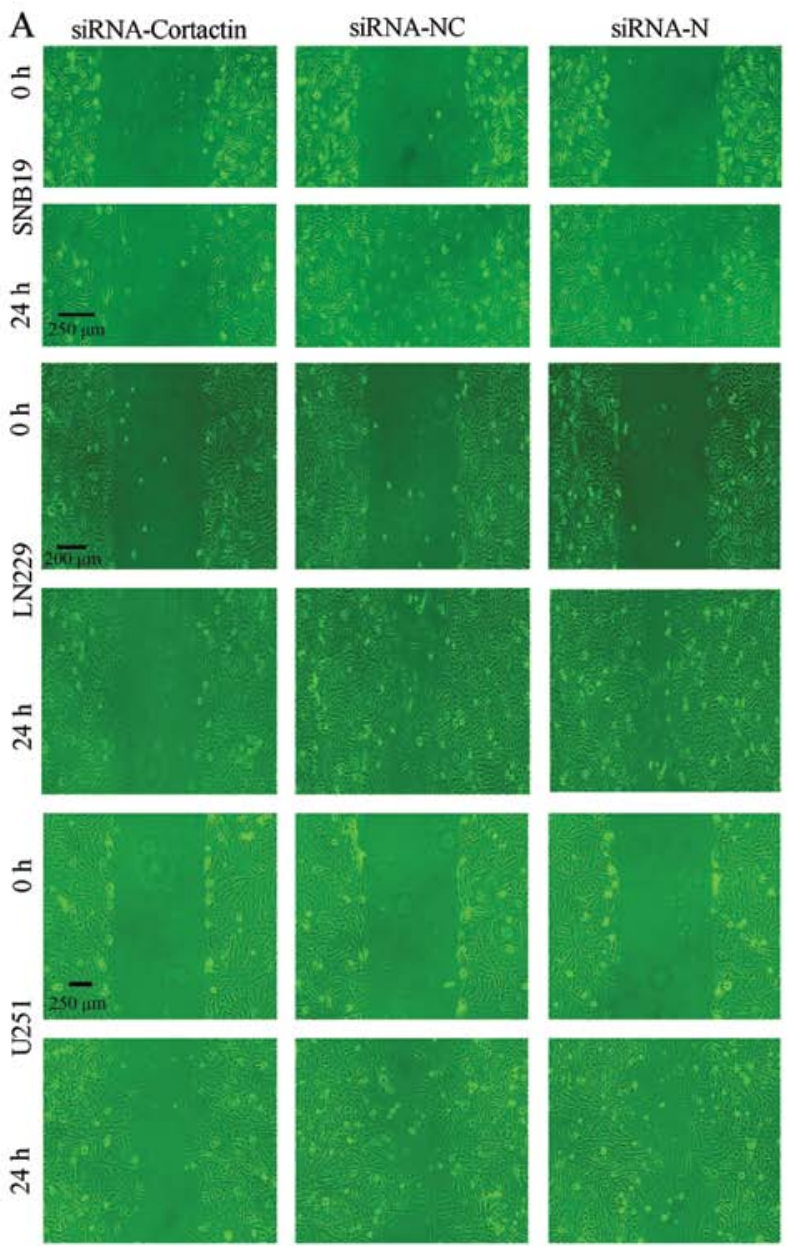

\section{B}

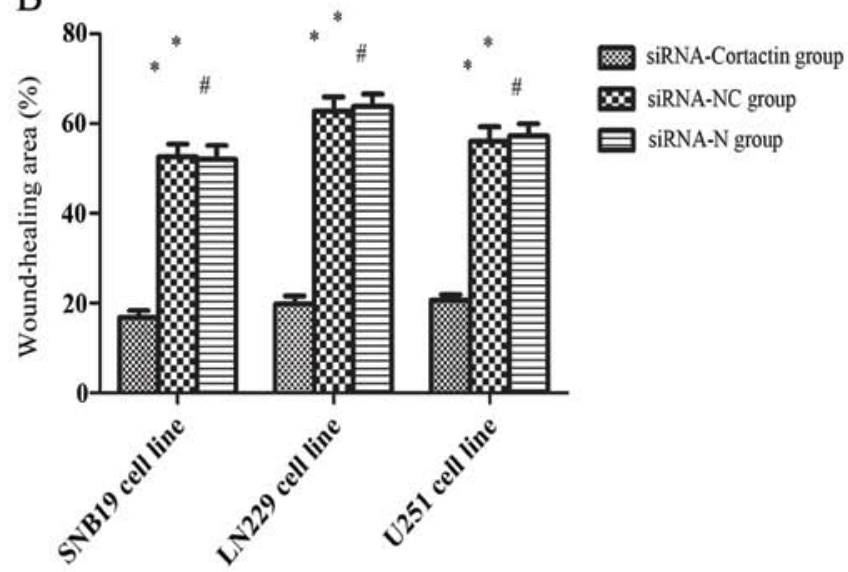

Figure 3. The migration ability of different treatment groups of glioma cells assessed by a wound-healing assay. After treatment with specific siRNA for $24 \mathrm{~h}$, wounds were created with a $200 \mu \mathrm{l}$ sterile pipette tip and the medium was then changed with serum-free medium. The cells were further cultivated for up to an additional $24 \mathrm{~h}$ and photographed. (A) Compared with the Cortactin-NC and Cortactin-N groups, the siRNA-cortactin group showed a wider wound area after wound generation for $24 \mathrm{~h}$. (B) Quantified data showed that specific siRNA for cortactin significantly inhibited glioma cell migration in vitro $\left({ }^{*} \mathrm{P}<0.05\right.$ and $\left.{ }^{\#} \mathrm{P}>0.05\right)$.

$57.20 \pm 2.76 \%$ in $\mathrm{U} 251$ cells $)(\mathrm{P}<0.05)$. These results suggested that inhibition of cortactin by siRNA effectively reduced the migration ability of glioma cells (Fig. 3A and B).

Part of the invasion cascade involves tumor cells attaching to and penetrating basement membranes. Therefore, basement
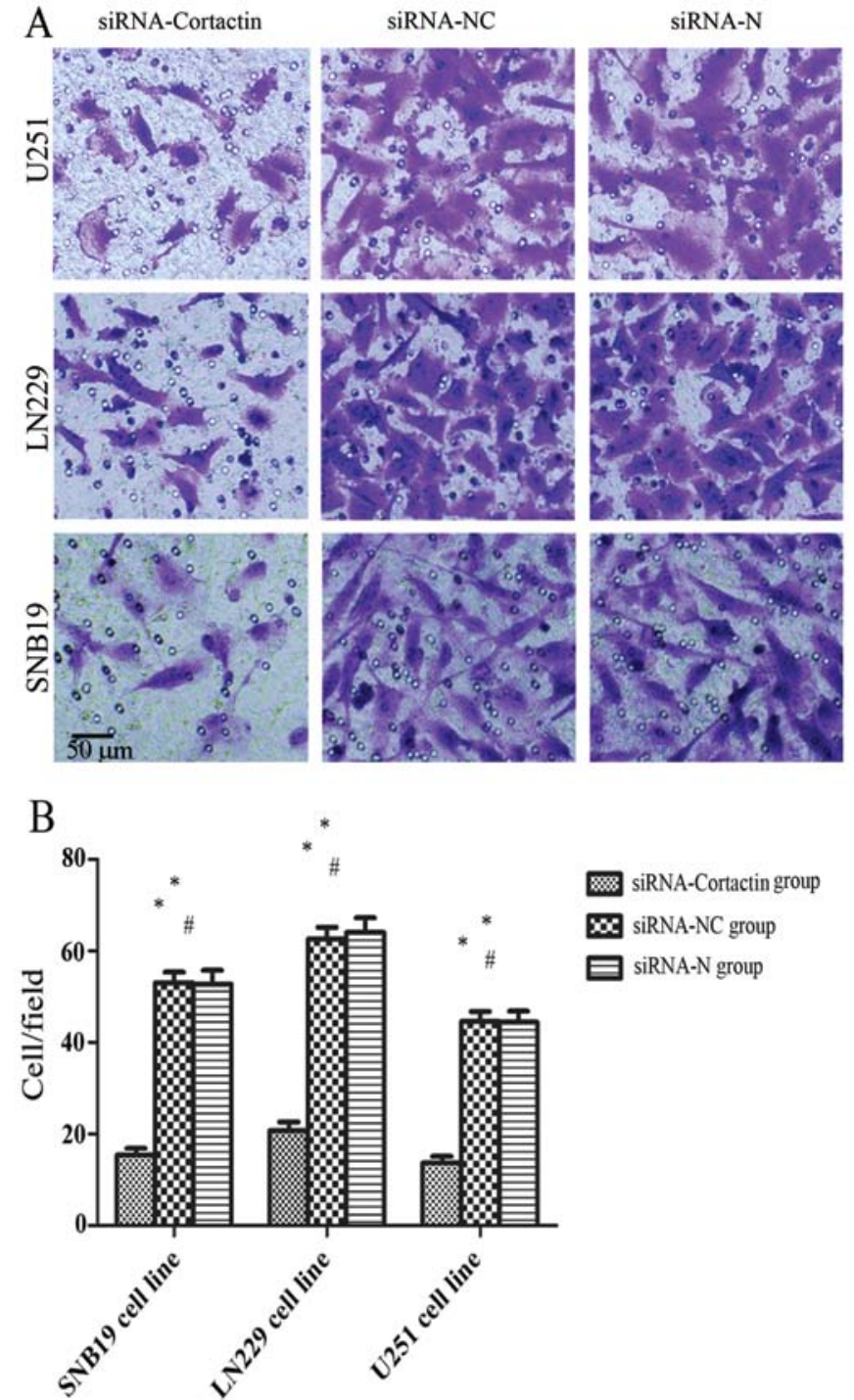

Figure 4. Knockdown of cortactin inhibits glioma cell invasion in vitro. After treatment for $24 \mathrm{~h}$, the Transwell invasion assay was performed. Following incubation for $48 \mathrm{~h}$, the cells that had migrated through the Matrigel were stained by crystal violet and photographed. (A) Representative images of glioma cells in the Millipore Matrigel-coated invasion chambers. (B) Quantified data show that specific siRNA significantly inhibited the invasion of glioma cells in vitro $\left({ }^{*} \mathrm{P}<0.05\right.$ and $\left.{ }^{\#} \mathrm{P}>0.05\right)$.

membranes are critical barriers to the passage of disseminating tumor cells. The Transwell chamber with Matrigel has been used to assess the invasive ability of tumor cells. Since cell migration and invasion are critical properties for the diffuse growth of glioma, we investigated the role of inhibition of cortactin by siRNA on tumor cell invasion using the Transwell invasion assay. The number of cells migrating through the membrane of the siRNA-cortactin group $(15.4 \pm 1.43$ in SNB19, $20.80 \pm 1.85$ in LN229 and $13.60 \pm 1.50 \%$ in U251 cells) was less than that in the siRNA$\mathrm{NC}$ group $(53.00 \pm 2.39$ in SNB19, 62.60 2.58 in LN229 and $44.60 \pm 2.16 \%$ in U251 cells) and the siRNA-N group $(52.80 \pm 3.94$ in SNB19 cells, $64.00 \pm 3.24 \%)$ in LN229 cells, $(44.40 \pm 2.44 \%$ in $\mathrm{U} 251$ cells $)(\mathrm{P}<0.05)$. The invasion of $\mathrm{U} 251$, LN229 and SNB19 glioma cells across the Transwell chamber were significantly impaired by siRNA compared to the siRNA-NC and siRNA-N groups (Fig. 4A and $\mathrm{B})(\mathrm{P}<0.05)$. 


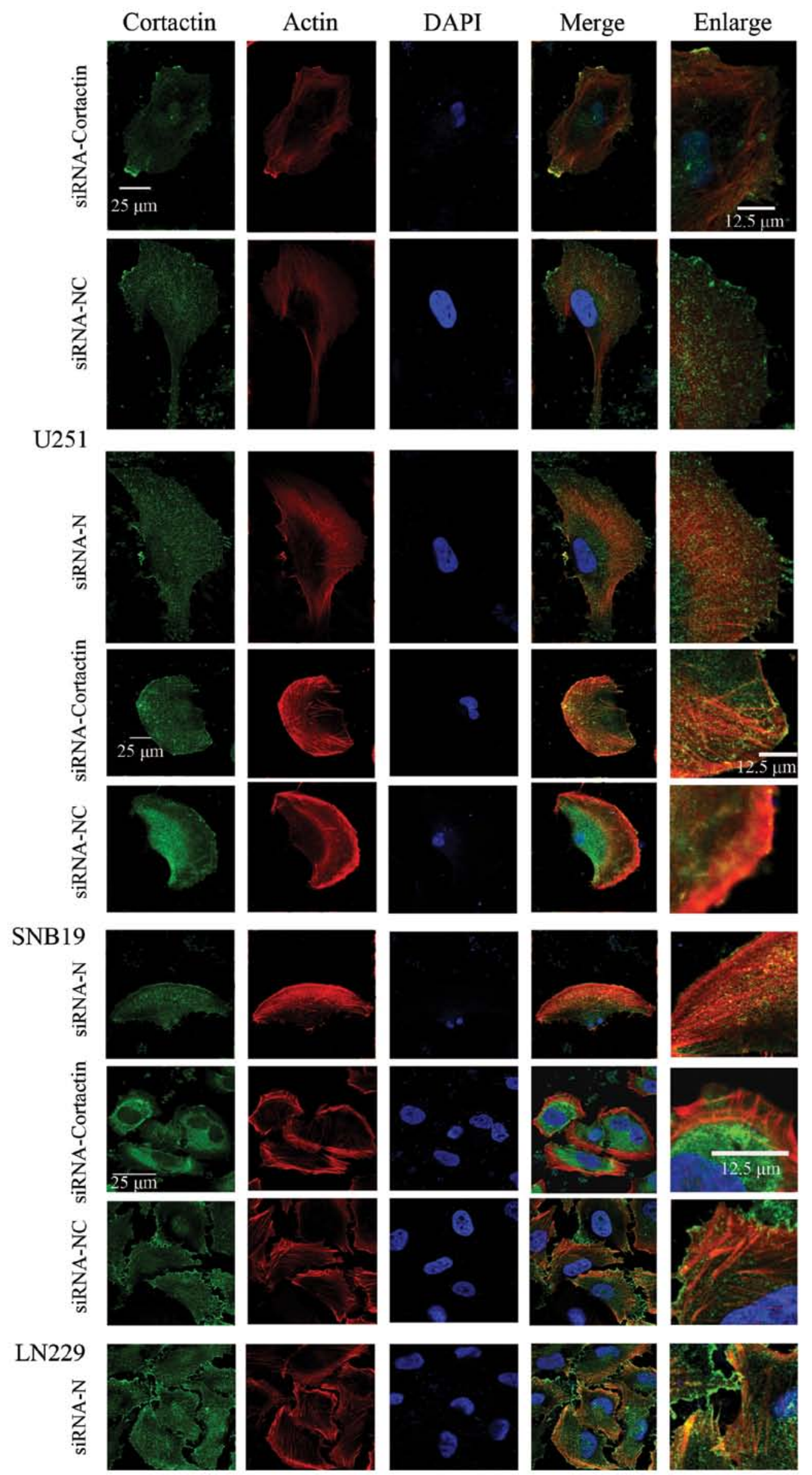

Figure 5. Knockdown of cortactin inhibits the formation of lamellipodia in glioma cells. After treatment with siRNA for $24 \mathrm{~h}$, the glioma cells of the three treatment groups were grown on glass coverslips for $24 \mathrm{~h}$ and then IF was performed. The images show that lamellipodia were smaller following treatment with specific siRNA. Images were captured using a confocal microscope. Actin (red), Cortactin (green) and nuclei (blue). IF, immunofluorescence. 
Knocked down expression of cortactin alters the morphology of glioma cells. To assess the inhibition of cortactin by siRNA on the lamellipodia of glioma cells, glioma cells in the various treated groups were stained for actin with rhodamine phalloidin, while IF was used for cortactin and contactin antibody and DAPI, respectively, for the cell nucleus. The result showed cortactin was localized in the F actin-enriched area. The lamellipodia were smaller in the cells treated with siRNA specific to cortactin compared to the other two groups (Fig. 5). This result explains that cortactin plays a key role in the formation of lamellipodia in glioma cells.

Knocked down expression of cortactin reduces the persistence time of lamellipodia of glioma cells. To examine the persistence time of lamellipodia after the expression of cortactin in glioma cells was knocked down, glioma cells in each treated group were placed under an inverted microscope to observe the transformation of lamellipodia at 0,15 and 30 min for the three cell lines. The result showed that the size of lamellipodia was smaller ( $0 \mathrm{~min})$ and the persistence time was reduced (15 and $30 \mathrm{~min}$ ) in the cells of the siRNA-cortactin group than the remaining two groups (Fig. 6). This result showed that cortactin maintained the persistence time of lamellipodia in glioma cells.

Cortactin and the Arp2/3 complex are co-localized in glioma cells. To investigate the relationship between cortactin and the Arp2/3, IF of cortactin and the Arp2/3 was detected in U251, LN229 and SNB19 human glioma cells. Glioma cells were stained with cortactin antibody, p34-Arc subunit antibody specific for the Arp2/3 complex, rhodamine phalloidin for actin filaments and DAPI for nucleus. The result showed that cortactin co-localized with the actin-related protein Arp2/3 complex (Fig. 8) at sites of actin polymerization within the lamellipodia (Fig. 7).

\section{Discussion}

A hallmark of malignant gliomas is their ability to disperse through neural tissue $(24,25)$. Cortactin plays a positive role in the migration and invasion of many other tumors $(17,26-$ 29), however, its role in gliomas remain to be determined. In the initial phase of the present study, we explored the expression of cortactin in different grade gliomas and non-tumor brain tissues. We found that cortactin was expressed weakly in non-tumor brain tissues, but strongly expressed in gliomas and the expression level of cortactin was positively correlated with the malignancy of gliomas. This result showed that cortactin plays a key role in gliomas and can clarify highergrade glioma infiltration into the surrounding brain tissue. The result also encouraged us to investigate the mechanism of cortactin in glioma motility. Studies were performed in three human glioma cell lines in vitro and we observed the effect of the reduction of cortactin in the migration and invasion of glioma cells. Following treatment with specific cortactin siRNA, the expression of cortactin was decreased at the transcription and translation level in glioma cells. The wound-healing and Transwell invasion assays, respectively, revealed that migration and invasion was decreased markedly after glioma cells were treated. Therefore it suggests that the possible mechanism of the above results is the inhibition of

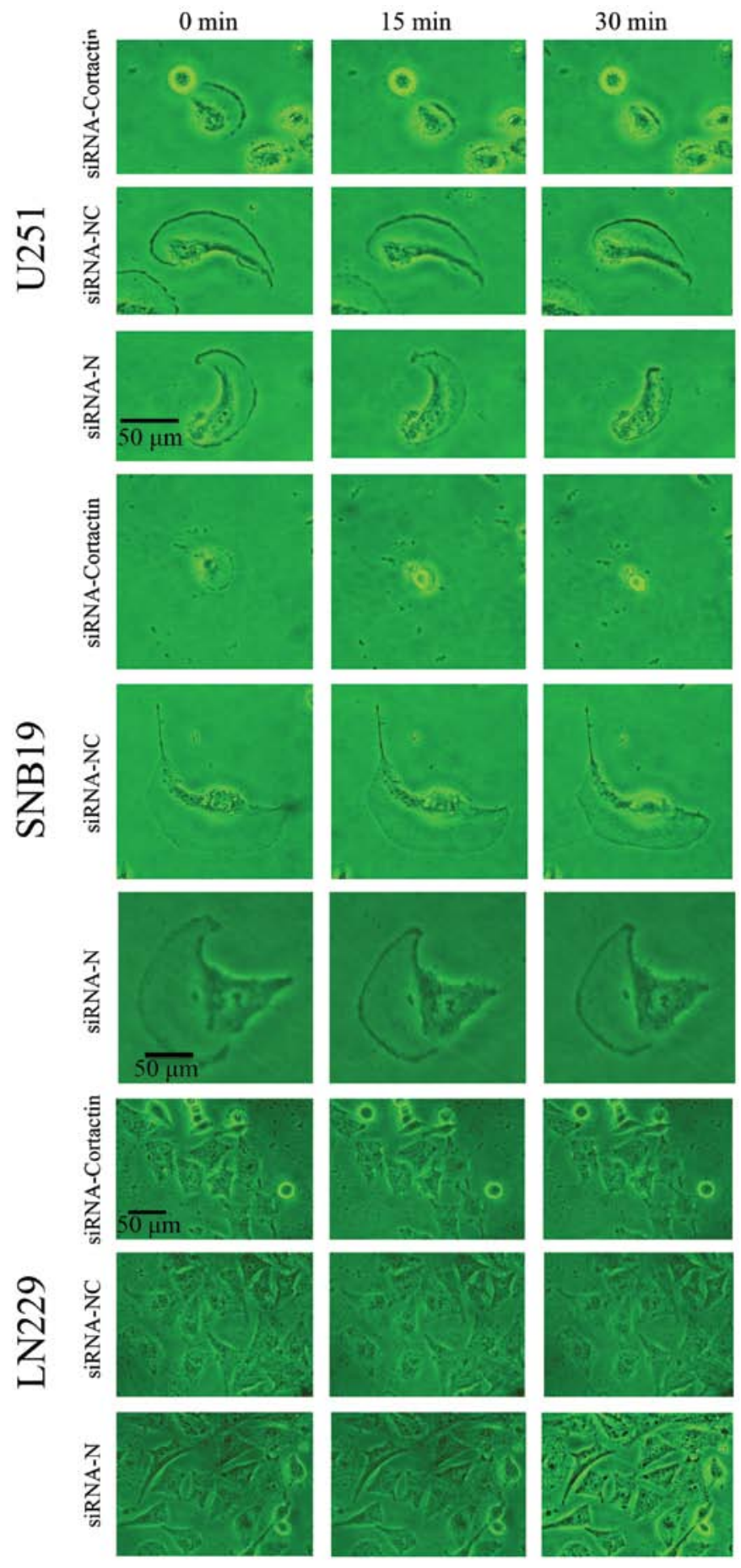

Figure 6. Knockdown of cortactin reduces the persistence time of lamellipodia in glioma cells. After treatment with specific siRNA $24 \mathrm{~h}$, the three treatment groups of each glioma cell line were placed under an inverted microscope to observe the transformation of lamellipodia and imaged at 0,15 and $30 \mathrm{~min}$ The result shows that the lamellipodia were smaller $(0 \mathrm{~min})$ and the persistence time was reduced (15 and $30 \mathrm{~min}$ ) after silencing the expression of cortactin using siRNA compared to the remaining two groups.

cortactin, which reduces its ability to regulate lamellipodia formation in glioma cells.

Cortactin promotes cell motility by regulating the characteristics of lamellipodia including their stability or persistence, and actin dynamics within the lamellipodia (16). The main migration movement pattern of glioma cells is 

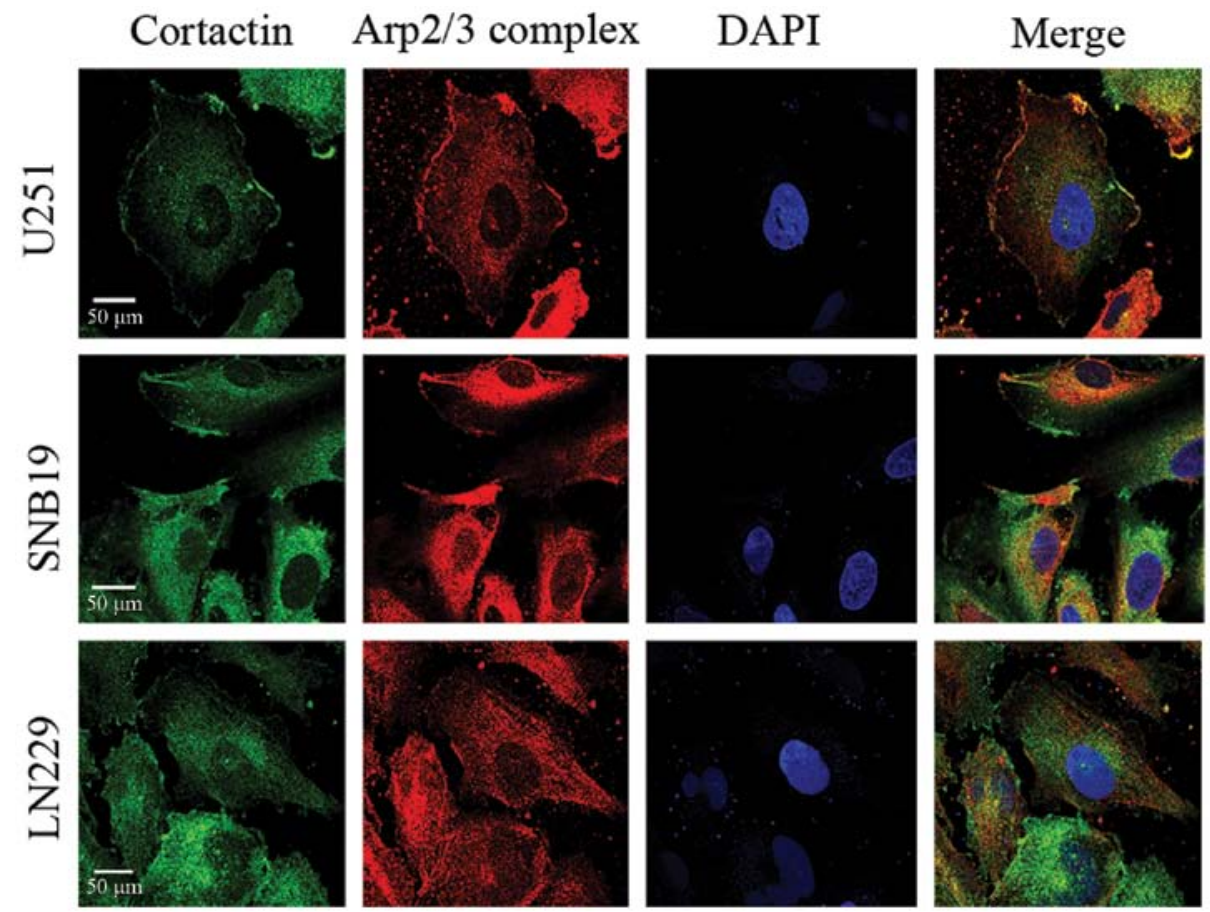

Figure 7. The distribution of actin, cortactin and Arp2/3 complex in glioma cells. Phalloidin staining of cells showed typical actin organization with F-actin enriched at the lamellipodia leading edge. Furthermore, staining with the anti-cortactin and the anti-p34 antibody confirmed that cortactin and the Arp2/3 complex located in the region had rich actin in glioma cells.

interstitial movement, which has four continuous processes: tumor cells detach from the solid tumors, tumor cell adhesion to extracellular matrix (ECM), the degradation of ECM and tumor cells movement and contraction (4,29,30). Lamellipodia is the organization of membrane domains and the primary sites of actin incorporation, and plays an important role in cell movement $(6,18)$. To some extent, regulation of the formation and persistence of lamellipodia, results in the restriction of the interstitial movement of glioma cells. For this reason, we stained glioma cells using IF and observed the variation of lamellipodia in glioma cells after the down regulation of cortactin. The result showed that the size and persistence time of lamellipodia was reduced. These findings suggest a reduction of cortactin can decrease the formation of lamellipodia and the movement ability of glioma cells.

Cortactin and the Arp2/3 complex are closely associated with the regulation of cell motility (8). The Arp $2 / 3$ complex is an evolutionarily conserved actin nucleation factor localized in the lamellipodia. The dendritic nucleation model has been rigorously evaluated in several computational studies experimental studies demonstrating a critical role for Arp2/3 in the generation of protrusive actin structures and cell motility $(9,31)$. Previous findings have shown that the Arp2/3 complex plays a key role in the regulation of lamellipodia in glioma cells (13). In the present study, we found that cortactin is pivotal in the formation and persistence of lamellipodia, the former of which is inconsistent with findings of previous studies $(20,21)$. The decrease of the ability to regulate other related proteins, especially the Arp2/3 complex, was the main reason for the result. On the other hand, the interaction between cortacin and actin was also decreased following the inhibition of cortactin. In another result of IF, it was found that cortactin, actin and the
Arp2/3 complex were located in the membrane surrounding the site where lamellipodia formed. This result suggests that, actin as the material of lamellipodia is regulated by many molecules, including cortactin and the Arp2/3 complex. Additionally, consistent with other studies, cortactin and the Arp2/3 complex play a role in actin polymerization, and the two proteins may exhibit collaborative action in glioma cells. To confirm the result, we used the double staining of cortactin and the Arp2/3 complex. The result showed that cortactin and the Arp2/3 complex were co-localized in the front of glioma cells, which explains our results.

The main reason gliomas are incurable is the wide dissemination of these cells as opposed to the anti-glioma invasion (34). Cilengitide, an inhibitor of $\alpha v \beta 3$ and $\alpha v \beta 5$ integrin receptor did not affect the promotion of the median survival rate in patients with malignant gliomas (33-35). Cell movement is important to identifying treatment for various types of cancer. Cortactin and the Arp2/3 complex as the main molecules associated with cell movement, have been demonstrated to play a key role in glioma cell migration and invasion $(26,38)$. Thus, they may serve as new targets and contribute to the identification of appropriate anti-glioma treatment.

In summary, cortactin plays a crucial role in the migration and invasion of glioma. Our results indicate that cortactin promotes the motility of glioma cells by adjusting lamellipodia and this process requires the combination of cortactin and the Arp2/3 complex. Future studies should be conducted to examine cortactin promoting lamellipodia formation in vivo and the interaction with its binding proteins such as N-WASP and cofilin $(20,36)$. Investigation of cortactin with regard to migration and invasion may lead to identification of a treatment for inhibiting glioma infiltrative growth. 

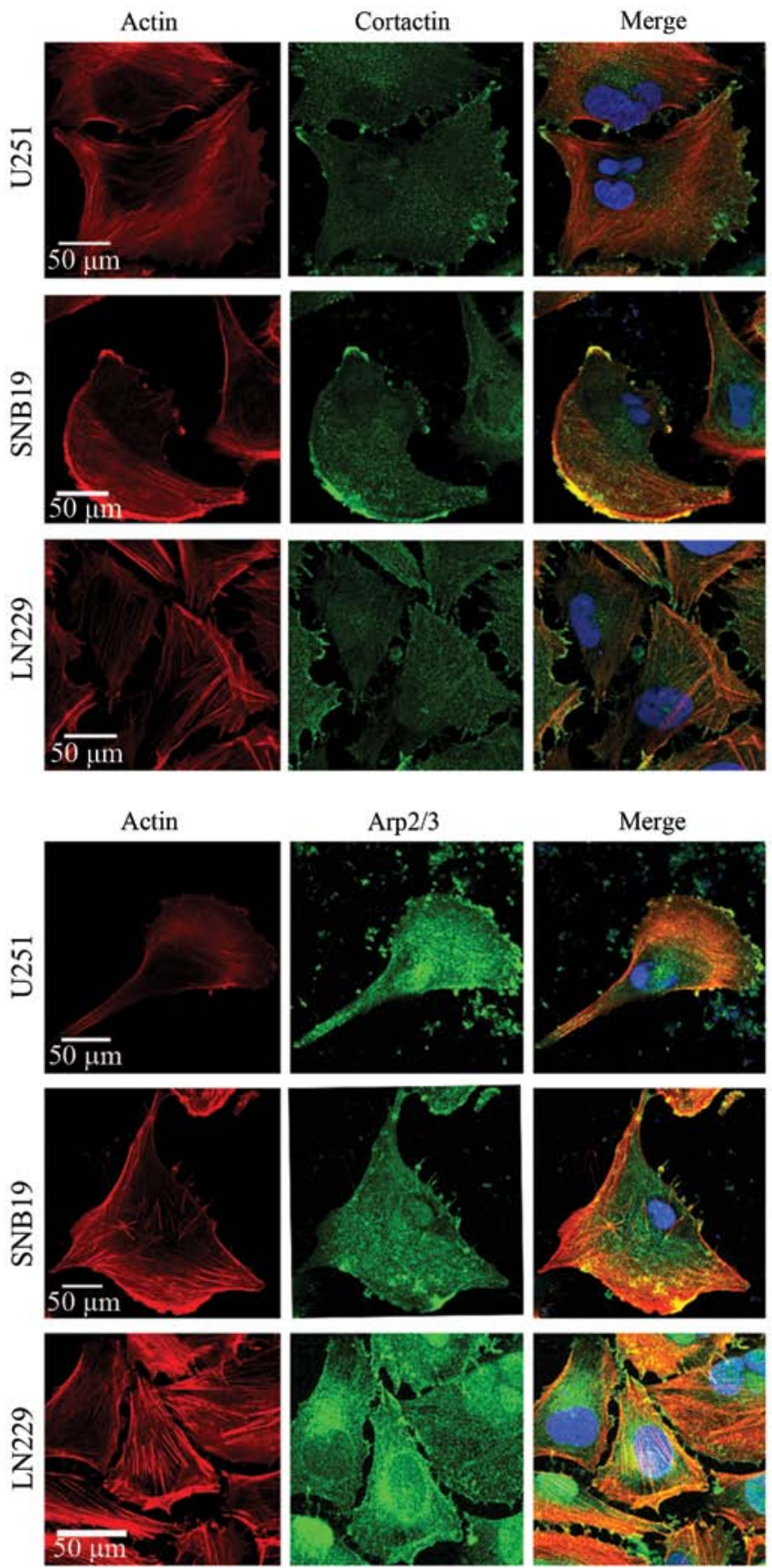

Figure 8. Co-localization of cortactin and Arp2/3 complex in glioma cells. The anti-cortactin and the anti-p34 antibody staining confirmed that the location of cortactin and the Arp2/3 complex are analogous. Cortactin and the Arp2/3 complex are located in the cell cytoplasm, especially the cell membrane.

\section{Acknowledgements}

The present study was supported by a grant from the National Natural Science Foundation of China (no. 81272782) and the Research Fund for the Doctoral program of Higher Education of China (no.20131202110006).

\section{References}

1. Alqudah MA, Agarwal S, Al-Keilani MS, Sibenaller ZA, Ryken TC and Assem M: NOTCH3 is a prognostic factor that promotes glioma cell proliferation, migration and invasion via activation of CCND1 and EGFR. PLoS One 8: e77299, 2013.
2. Nakada M, Nakada S, Demuth T, Tran NL, Hoelzinger DB and Berens ME: Molecular targets of glioma invasion. Cell Mol Life Sci 64: 458-478, 2007.

3. Sciumè G, Santoni A and Bernardini G: Chemokines and glioma: Invasion and more. J Neuroimmunol 224: 8-12, 2010.

4. Agudelo-Garcia PA, De Jesus JK, Williams SP, Nowicki MO, Chiocca EA, Liyanarachchi S, Li PK, Lannutti JJ, Johnson JK, Lawler SE, et al: Glioma cell migration on three-dimensional nanofiber scaffolds is regulated by substrate topography and abolished by inhibition of STAT3 signaling. Neoplasia 13: 831-840, 2011.

5. Godlewski J, Bronisz A, Nowicki MO, Chiocca EA and Lawler S: microRNA-451: A conditional switch controlling glioma cell proliferation and migration. Cell Cycle 9: 2742-2748, 2010.

6. Small JV, Stradal T, Vignal E and Rottner K: The lamellipodium: Where motility begins. Trends Cell Biol 12: 112-120, 2002.

7. Yamaguchi $\mathrm{H}$ and Condeelis $\mathrm{J}$ : Regulation of the actin cytoskeleton in cancer cell migration and invasion. Biochim Biophys Acta 1773: 642-652, 2007.

8. Wu C, Asokan SB, Berginski ME, Haynes EM, Sharpless NE, Griffith JD, Gomez SM and Bear JE: Arp2/3 is critical for lamellipodia and response to extracellular matrix cues but is dispensable for chemotaxis. Cell 148: 973-987, 2012.

9. Suraneni P, Rubinstein B, Unruh JR, Durnin M, Hanein D and Li R: The Arp2/3 complex is required for lamellipodia extension and directional fibroblast cell migration. J Cell Biol 197: 239-251, 2012.

10. Goley ED and Welch MD: The ARP2/3 complex: An actin nucleator comes of age. Nat Rev Mol Cell Biol 7: 713-726, 2006

11. Koestler SA, Steffen A, Nemethova M, Winterhoff M, Luo N, Holleboom JM, Krupp J, Jacob S, Vinzenz M, Schur F, et al: Arp2/3 complex is essential for actin network treadmilling as well as for targeting of capping protein and cofilin. Mol Biol Cell 24: 2861-2875, 2013

12. Iwaya K, Norio K and Mukai K: Coexpression of Arp2 and WAVE2 predicts poor outcome in invasive breast carcinoma. Mod Pathol 20: 339-343, 2007.

13. Liu Z, Yang X, Chen C, Liu B, Ren B, Wang L, Zhao K, Yu S and Ming H: Expression of the Arp2/3 complex in human gliomas and its role in the migration and invasion of glioma cells. Oncol Rep 30: 2127-2136, 2013.

14. Croucher DR, Rickwood D, Tactacan CM, Musgrove EA and Daly RJ: Cortactin modulates RhoA activation and expression of Cip/Kip cyclin-dependent kinase inhibitors to promote cell cycle progression in 11q13-amplified head and neck squamous cell carcinoma cells. Mol Cell Biol 30: 5057-5070, 2010.

15. MacGrath SM and Koleske AJ: Cortactin in cell migration and cancer at a glance. J Cell Sci 125: 1621-1626, 2012.

16. Weaver AM, Karginov AV, Kinley AW, Weed SA, Li Y, Parsons JT and Cooper JA: Cortactin promotes and stabilizes Arp2/3-induced actin filament network formation. Curr Biol 11: 370-374, 2001.

17. Weed SA, Karginov AV, Schafer DA, Weaver AM, Kinley AW, Cooper JA and Parsons JT: Cortactin localization to sites of actin assembly in lamellipodia requires interactions with F-actin and the Arp2/3 complex. J Cell Biol: 29-40, 2000.

18. Bryce NS, Clark ES, Leysath JL, Currie JD, Webb DJ and Weaver AM: Cortactin promotes cell motility by enhancing lamellipodial persistence. Curr Biol 15: 1276-1285, 2005.

19. Dedes KJ, Lopez-Garcia MA, Geyer FC, Lambros MB, Savage K, Vatcheva R, Wilkerson P, Wetterskog D, Lacroix-Triki M, Natrajan R, et al: Cortactin gene amplification and expression in breast cancer: A chromogenic in situ hybridisation and immunohistochemical study. Breast Cancer Res Treat 124: 653-666, 2010.

20. Desmarais V, Yamaguchi H, Oser M, Soon L, Mouneimne G, Sarmiento C, Eddy R and Condeelis J: N-WASP and cortactin are involved in invadopodium-dependent chemotaxis to EGF in breast tumor cells. Cell Motil Cytoskeleton 66: 303-316, 2009.

21. Hofman P, Butori C, Havet K, Hofman V, Selva E, Guevara N, Santini J and Van Obberghen-Schilling E: Prognostic significance of cortactin levels in head and neck squamous cell carcinoma: Comparison with epidermal growth factor receptor status. $\mathrm{Br} \mathrm{J}$ Cancer 98: 956-964, 2008.

22. Yamada S, Yanamoto S, Kawasaki G, Mizuno A and Nemoto TK: Overexpression of cortactin increases invasion potential in oral squamous cell carcinoma. Pathol Oncol Res 16: 523-531, 2010.

23. Perrin BJ, Amann KJ and Huttenlocher A: Proteolysis of cortactin by calpain regulates membrane protrusion during cell migration. Mol Biol Cell 17: 239-250, 2006. 
24. Giese A, Bjerkvig R, Berens ME and Westphal M: Cost of migration: Invasion of malignant gliomas and implications for treatment. J Clin Oncol 21: 1624-1636, 2003.

25. Westermark B: Glioblastoma - a moving target. Ups J Med Sci 117: 251-256, 2012.

26. Weaver AM: Cortactin in tumor invasiveness. Cancer Lett 265 $157-166,2008$.

27. Tehrani S, Faccio R, Chandrasekar I, Ross FP and Cooper JA: Cortactin has an essential and specific role in osteoclast actin assembly. Mol Biol Cell 17: 2882-2895, 2006.

28. Hou H, Chen W, Zhao L, Zuo Q, Zhang G, Zhang X, Wang H, Gong $\mathrm{H}$, Li X, Wang M, et al: Cortactin is associated with tumour progression and poor prognosis in prostate cancer and SIRT2 other than HADC6 may work as facilitator in situ. J Clin Pathol 65: 1088-1096, 2012.

29. Jovčevska I, Kočevar N and Komel R: Glioma and glioblastoma - how much do we (not) know? Mol Clin Oncol 1: 935-941, 2013.

30. Rao JS: Molecular mechanisms of glioma invasiveness: The role of proteases. Nat Rev Cancer 3: 489-501, 2003.

31. Pfaendtner J, Volkmann N, Hanein D, Dalhaimer P, Pollard TD and Voth GA: Key structural features of the actin filament Arp2/3 complex branch junction revealed by molecular simulation. J Mol Biol 416: 148-161, 2012.
32. Chang L, Su J, Jia X and Ren H: Treating malignant glioma in Chinese patients: Update on temozolomide. Onco Targets Ther 7: 235-244, 2014

33. Reardon DA, Fink KL, Mikkelsen T, Cloughesy TF, O'Neill A, Plotkin S, Glantz M, Ravin P, Raizer JJ, Rich KM, et al: Randomized phase II study of cilengitide, an integrin-targeting arginine-glycine-aspartic acid peptide, in recurrent glioblastoma multiforme. J Clin Oncol 26: 5610-5617, 2008.

34. Eisele G, Wick A, Eisele A-C, Clément PM, Tonn J, Tabatabai G, Ochsenbein A, Schlegel U, Neyns B, Krex D, et al: Cilengitide treatment of newly diagnosed glioblastoma patients does not alter patterns of progression. J Neurooncol 117: 141-145, 2014.

35. Cheng NC, van Zandwijk N and Reid G: Cilengitide inhibits attachment and invasion of malignant pleural mesothelioma cells through antagonism of integrins $\alpha v \beta 3$ and $\alpha v \beta 5$. PLoS One 9: e90374, 2014.

36. Yu X, Zech T, McDonald L, Gonzalez EG, Li A, Macpherson I, Schwarz JP, Spence H, Futó K, Timpson P, et al: N-WASP coordinates the delivery and F-actin-mediated capture of MT1-MMP at invasive pseudopods. J Cell Biol 199: 527-544, 2012. 